\title{
Development of Glutamic Acid Decarboxylase Immunoreactivity in the Cat's Lateral Geniculate Nucleus
}

\author{
Sandra L. Shotwell, ${ }^{1}$ Carla J. Shatz, and Marla B. Luskin ${ }^{2}$ \\ Department of Neurobiology, Stanford University School of Medicine, Stanford, California 94305
}

The development of glutamic acid decarboxylase (GAD) immunoreactivity in the cat's dorsal LGN was studied during fetal and postnatal life. In the adult, inhibitory interactions within the LGN are known to be mediated by GABA. Here we have used an antiserum to GAD, the rate-limiting synthetic enzyme for GABA, to examine the development of the anatomical substrate for this inhibitory system.

The pattern of immunostaining observed in the adult cat LGN was similar to that reported by Fitzpatrick et al. (1984), with heavily stained somata and proximal dendrites located within the LGN layers and the adjacent perigeniculate nucleus (PGN). The LGN also contained a complex array of terminal staining. In development, specific staining was seen about 2 weeks before birth and was confined to PGN somata and, to a lesser extent, to somata located in the future ventral C-layers. A similar pattern of immunostaining was seen using GABA antiserum. Not until birth did the A-layers of the LGN show appreciable staining of both somata and terminals; however, even then the pattern of immunostaining was far from mature. Furthermore, excessive numbers of PGN neurons appeared to stain. By 5 weeks after birth, the intensity of both soma and terminal staining within the A-layers of the LGN increased substantially relative to that of the PGN and ventral C-layers. The first glomerular clusters of terminal staining could also be seen, and the number of stained PGN neurons had diminished to levels similar to those seen in the adult. The pattern of immunostaining was almost adultlike by 2 months after birth, except within the C-complex, where the staining did not yet show the distinct difference in staining intensity present in the adult between dorsal layer $C$ and ventral layers $C 1$ and $C 2$. The final adult pattern of GAD immunoreactivity appeared by 3 months after birth.

These results suggest that during fetal life the PGN and ventral C-layers of the LGN may supply the first source of GABAmediated inhibition to the nucleus, with the major portion of the inhibition supplied by intrinsic LGN neurons arising postnatally. Thus, PGN neurons may provide part of the anatomical substrate for the inhibitory interactions seen physiologically during late fetal development (Shatz and Kirkwood, 1984). Finally, the relatively late appearance of the adultlike pattern of

\footnotetext{
Received July 23, 1985; revised Sept. 19, 1985; accepted Oct. 3, 1985.

Special thanks to Drs. Donald Schmechel and Wolfgang Oertel for generously providing GAD antiserum. We are grateful to Drs. Peter Kirkwood, Peter Sargent, and David Sretavan for their useful criticisms of the manuscript; Mark Siegel for assistance with photography; and Jerold Chun, David Sretavan, and Mark Siegel for assistance with the fetal surgeries. Thanks also to Cecele Thomas for help with word processing. This research was supported by grants from the NSF (BNS 8317228), the NIH (EY 02858), the March of Dimes (C.J.S.), and NIH Grants EY 05632 (S.L.S.) and NS 07158 (M.B.L.)

Correspondence should be addressed to Dr. Shatz at the above address.

1 Present address: Stanford University, Office of Technology Licensing, 350 Cambridge Ave., Suite 250, Palo Alto, CA 94306

${ }_{2}^{2}$ Present address: Vision Center Laboratory, Salk Institute for Biological Studies, San Diego, CA 92138.
}

Copyright (C) 1986 Society for Neuroscience $0270-6474 / 86 / 051410-14 \$ 02.00 / 0$
GAD immunostaining suggests that intrageniculate inhibitory circuitry continues to develop well after birth.

In the mammalian CNS, inhibitory interactions play an important role in the shaping of neuronal response properties. For example, in the visual system, the directional selectivity of rabbit retinal ganglion cells (Ariel and Daw, 1982) and a subset of cat visual cortical neurons (Sillito, 1977) requires circuitry utilizing the inhibitory transmitter GABA. Within the thalamus, GABA-mediated inhibition also underlies other visual response properties such as the center-surround receptive field organization characteristic of neurons of the LGN (Sillito and Kemp, 1985). And, though neurons of the LGN are commonly known to receive powerful monosynaptic excitatory inputs from retinal ganglion cell axons of one eye only, they also receive substantial disynaptic inhibition from the other eye (Burke and Sefton, 1966; Dubin and Cleland, 1977; Kato et al., 1971; Lindstrom, 1982; Suzuki and Kato, 1966). Thus, binocular interactions within the LGN depend critically on inhibitory circuitry.

Physiological and anatomical studies indicate that LGN neurons in the cat receive their inhibition from at least two sources: intrinsic interneurons within the LGN proper (Dubin and Cleland, 1977; Famiglietti and Peters, 1972; Lindstrom, 1982; Singer and Bedworth, 1972); and extrinsic neurons located in the adjacent perigeniculate nucleus (PGN) (Ahlsen and Lindstrom, 1982; Dubin and Cleland, 1977; Friedlander et al., 1981; Lindstrom, 1982). Recently, further confirmation of the contribution of intrinsic and PGN neurons to inhibitory interactions within the LGN has come from immunohistochemical studies using an antibody directed against glutamic acid decarboxylase (GAD), the rate-limiting synthetic enzyme for GABA. Fitzpatrick and coworkers (1984) have demonstrated immunohistochemical staining for GAD within a distinct population of local circuit neurons within the LGN, and within essentially all neurons of the PGN (see also Montero and Singer, 1984a, b).

While it is clear that inhibitory input plays an important role in the functioning of the adult visual system, very little is known about the time course of development of inhibition or its role in the formation of connections. In a previous physiological study of the development of functional connections between retinal ganglion cells and LGN neurons, Shatz and Kirkwood (1984) found that inhibitory interactions could first be detected about 2 weeks prior to birth in the cat. Here, we have examined the anatomical basis for the development of the GABA inhibitory system in the cat's LGN. Using an immunohistochemical assay for GAD, we have found that GAD immunoreactivity also appears about 2 weeks before birth. However, immunostaining does not reach its adult pattern or intensity until 2-3 months postnatally. This developmental time frame correlates with the period during which the cat's visual system is susceptible to the effects of abnormal visual experience (Blakemore and Van Sluyters, 1974; Hubel and Wiesel, 1970), raising the possibility that the inhibitory LGN circuitry could contribute 
to developmental alterations in neuronal response properties seen during this period. Some of these results have been reported previously in abstract form (Shotwell et al., 1984).

\section{Materials and Methods}

This study includes results from 25 animals ranging in age from $\mathrm{cm}$ bryonic day 42 (E42) to adult (gestation is $65 \mathrm{~d}$ in the cat). Nine fetal animals were studied, along with six neonates less than 2 weeks old, eight animals between 2 weeks and 3 months old, and two adults. Fetuses were delivered by Cesarian section, using sterile surgical technique, from timed-pregnant female cats anesthetized with $1-2 \%$ halothane mixed with nitrous oxide $(0.5 \mathrm{liter} / \mathrm{min})$ and oxygen ( $1 \mathrm{liter} / \mathrm{min})$. Full details of the fetal surgical methods can be found in Shatz (1983). Postnatal animals were anesthetized for perfusion with $40 \mathrm{mg} / \mathrm{kg}$ Nembutal. All animals were perfused, and tissue processed according to a modification of the protocol described by Fitzpatrick et al. (1984).

\section{Fixation}

Animals were perfused transcardially with $100 \cdot \mathrm{mm}$ PBS $(50-550 \mathrm{ml}$, depending on age), $\mathrm{pH} 7.4$, at room temperature (KI). Next, fixation was initiated with $4 \%$ paraformaldehyde in $100 \mathrm{~mm}$ phosphate buffer (PB), pH 7.4 (150-700 ml). Finally, an equal volume of periodate/lysine/ paraformaldehyde (PLP) fixative in $75 \mathrm{~mm}$ PB, pH 6.5 (McLean and Nakane, 1974), was perfused. The brain was exposed by opening the cranium, postfixed for an additional $2 \mathrm{hr}$ at RT, and then stored overnight in PLP at $4^{\circ} \mathrm{C}$. The brain was embedded in a block of $4 \%$ agar to facilitate sectioning. Depending on age, $50-100 \mu \mathrm{m}$ thick sections were cut on a vibratome, collected in $20 \mathrm{~mm}$ PBS, pH 7.6, and stored at $4^{\circ} \mathrm{C}$ until use. For the fetal studies, vibratome sections were found to be superior to frozen section in histological preservation, and $100 \mu \mathrm{m}$ sections worked best for the youngest animals. In order to compensate for the developmental rotation of the LGN, horizontal sections were cut for fetal and neonatal animals, while coronal sections were cut for those animals older than postnatal day ( $\mathrm{P}) 1$ (for explanation, see Kalil, 1978; Shatz, 1983)

\section{Immunohistochemistry}

These studies were performed with GAD antiserum from sheep and normal sheep plasma (NSP), both generously provided by Drs. Donald Schmechel and Wolfgang Oertel (Oertel et al., 1981) and GABA antiserum from rabbit (Immuno Nuclear). For GAD immunohistochemistry, sections were incubated immediately after sectioning in $20 \mathrm{~mm}$ PBS, $10 \mathrm{~mm}$ lysine, $1 \%$ normal rabbit serum (NRS; Miles Laboratories or Colorado Serum Company) for $1 \mathrm{hr}$ at RT. Each section was incubated floating in a separate well with gentle agitation. Addition of $0.02-0.2 \%$ Triton X-100 in preliminary trials resulted in an increase in the levels of nonspecific staining. The examples presented here are from material processed without Triton X-100.

After $1 \mathrm{hr}$ of preincubation, sections were transferred to fresh wells containing the preincubation solution plus GAD antiserum or control serum (NSP) at a final dilution of 1:1000 or 1:2000. In each experiment, an additional control section was processed using the preincubation solution containing NRS only. Sections were incubated for $3 \mathrm{hr}$ at RT (agitating) and then overnight at $4^{\circ} \mathrm{C}$. The Vectastain ABC Kit (Vector $\mathrm{Lab}$, Inc.) was used for immunoperoxidase staining. Sections were placed in individual baskets to facilitate handling of tissue and washed $2 \times 15$ $\min$ in PBS at RT. Sections were transferred into biotinylated secondary antibody at twice the suggested concentration for $30 \mathrm{~min}$. Two $5 \mathrm{~min}$ PBS washes were followed by $1 \mathrm{hr}$ incubation in avidin-biotin-HRP complex. The sections were washed in PBS several times and then washed several times in $50 \mathrm{~mm}$ Tris- $\mathrm{HCl}$, pH 7.4, plus $0.9 \% \mathrm{NaCl}$ (TBS).

The diaminobenzidene (DAB; Sigma Chemicals) reaction was carried out at $0.5 \mathrm{mg} / \mathrm{ml} \mathrm{DAB}$ in TBS (filtered with a $0.8 \mu \mathrm{m}$ Millipore filter) plus $0.02 \%$ hydrogen perioxide for several minutes. The reaction was continued until staining intensity stabilized and no further increase in intensity could be detected by visual inspection under a dissecting microscopc. The reaction time varied between 10 and $30 \mathrm{~min}$, and was not correlated with the age of the animal. We chose to maximize the reactions rather than running them for a fixed time period for several reasons. One was to ensure detection of immunoreactivity at the early ages. Another was to compensate for possible staining variability between experiments that might arise from variations in technique such as fixation, sectioning, or reagents. As an additional control, in most experiments a section of adult cat LGN was incubated in parallel to serve as a positive control and as an aid in determining when staining intensity had stabilized. Following completion of the reaction, sections were washed extensively in TBS, then in PBS, and stored in PBS at $4^{\circ} \mathrm{C}$ for further processing.

In two experiments, at $\mathrm{E} 49$ and at postnatal day 50, GABA antiserum made in rabbit and NRS control were used at dilutions of 1:2000. One percent goat serum was used as the blocking serum in place of NRS in the subsequent processing steps described above.

Following immunostaining, sections were mounted on subbed slides, and selected sections were lightly counterstained with cresyl violet. Sections were coverslipped with Permount and examined and photographed using standard bright-field or Nomarski optics.

\section{Results}

As a preface to studying the development of GAD immunoreactivity in the cat's LGN, we examined the pattern of immunostaining in several adult animals. The results presented in Figure 1 show a pattern of staining similar to that found by Fitzpatrick et al. (1984). As expected, all subdivisions of the LGN (layers A, Al, and the C-complex), the medial interlaminar nucleus (MIN), and the PGN contain GAD-positive terminals and somata (see Fig. $1 A$ ). The ventral geniculate nucleus (VGN) contains GAD-positive terminals only. The optic tract is free of stain. Essentially all PGN neurons exhibit rich staining within somata and proximal dendrites (Fig. $1 B$ ) (Fil_patrick el al., 1984; Montero and Singer, 1984a). Within the LGN proper, a subset of neurons are GAD-positive, and punctate terminal staining often is seen in clusters, particularly in the A-layers (Fig. 1C). Thus, the adult GABA inhibitory system includes these stained elements, which have been described in detail by Fitzpatrick el al. (1984).

\section{Prenatal development}

The pattern of immunostaining in all the fetal animals studied is very different from that seen in the adult. For example, at E56 the PGN exhibits distinct staining of numerous somata, as well as dendritic and possibly terminal staining (Fig. 2, $A$ and $B$ ). In contrast, relatively little soma staining can be seen within the LGN proper. Within the future A-layers [Fig. $2 A:(\mathrm{A})$ ], staining is very faint. Staining is scen occasionally in somata, but the majority is confined to radially oriented processes. Many of these probably are dendrites, because some can be seen to emanate from a faintly stained soma (see Fig. $2 C$, right). However, without further study we cannot rule out the possibility that some of these processes may be ingrowing axons. Within the future C-layers, definite immunostaining is found in the region closest to the optic tract (the future ventral C-layers). A small number of stained somata with processes oriented parallel to the optic tract lie within a fine dusting of terminal staining (Fig. $2 E)$.

We believe the pattern of immunostaining at E56 is specific because it did not appear in sections reacted with control serum. For example, Figure $2 D$ shows a region of future layer A comparable to that shown in Figure $2 C$, but taken from a control section incubated with NSP. Virtually no staining is present. However, the optic tracts of both control sections (not shown) and sections reacted with GAD antiserum were stained. Consequently, the presence of optic tract staining in control sections, though usually fainter than that seen with GAD antiserum, suggests that staining in this region may be nonspecific.

The earliest age at which we obtained reliable staining was E49. (In one younger animal, E42, immunostaining with the primary antibody was indistinguishable from that of controls.) By E49, all the neurons of the LGN have been generated (Hickey and Hitchcock, 1984; Shatz, 1981), and a majority have completed their migration and assumed a final position within the nucleus (C. J. Shatz and S. J. Eng, unpublished observations). Also at this age, many retinal ganglion cell axons have invaded the LGN and are beginning to sort out into eye-specific layers 


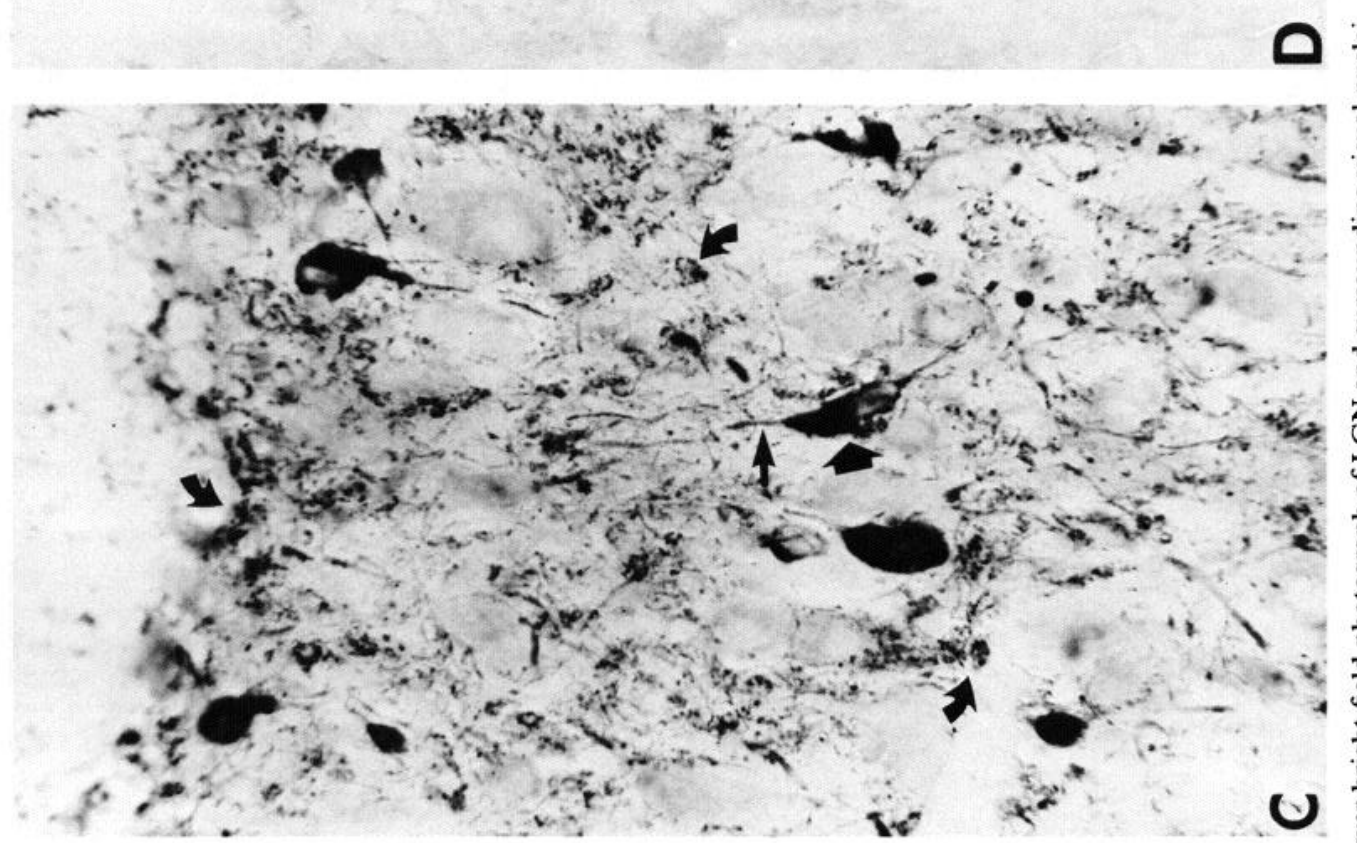

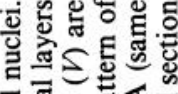

적 을

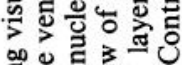

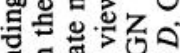

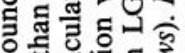

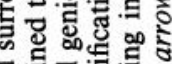

돓

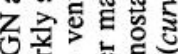

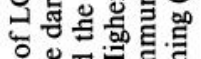

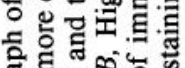

列.

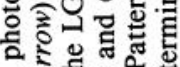

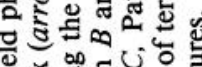

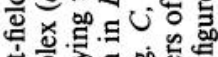

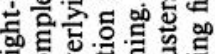

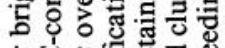

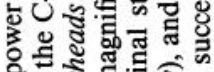

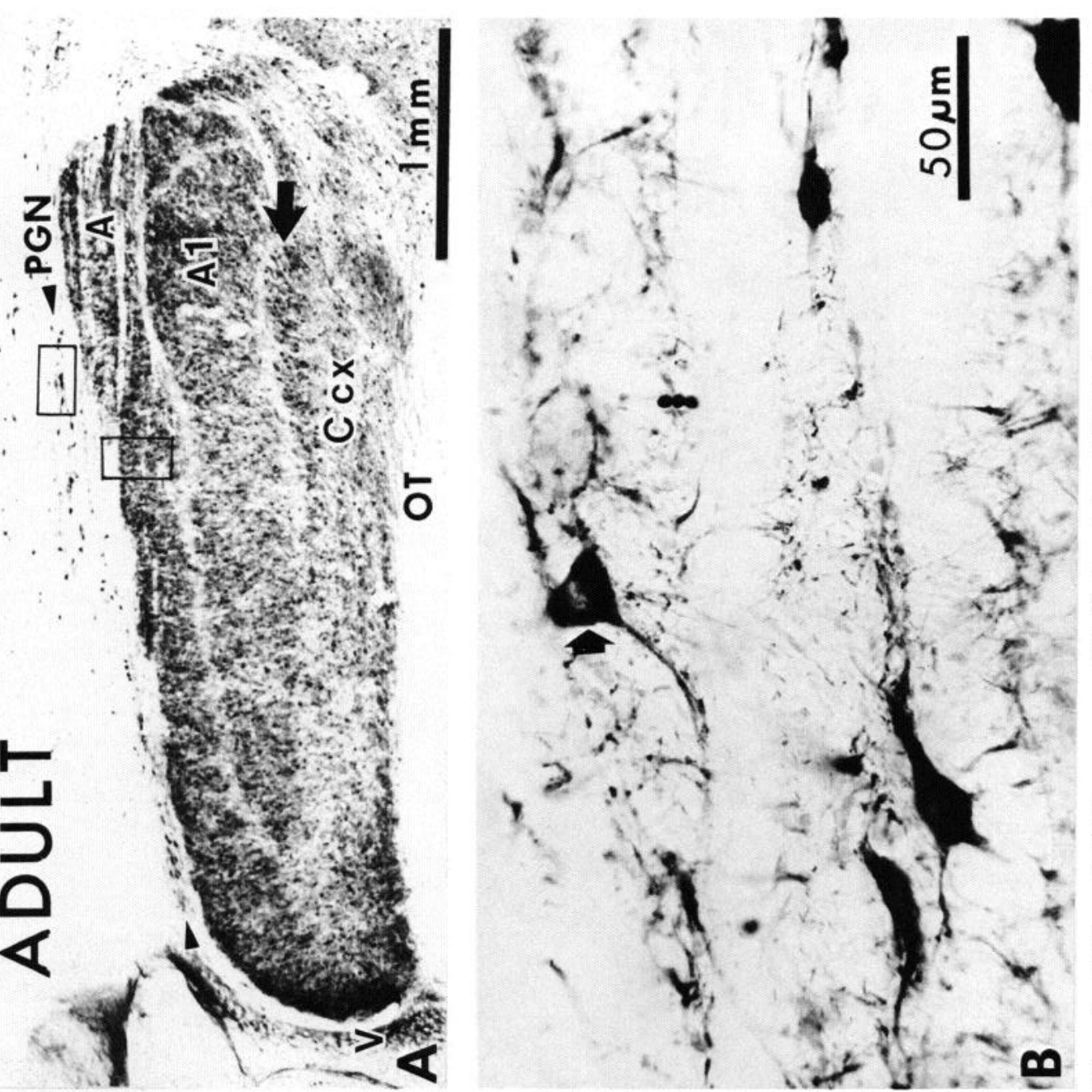

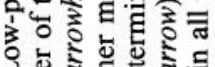

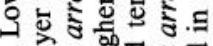

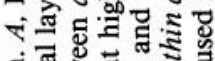

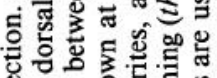
造㐘

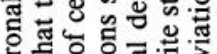

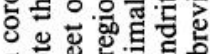
쿤동

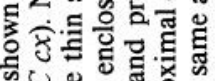
Z记

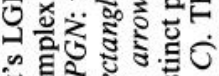

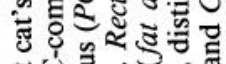
可记 을

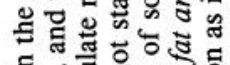

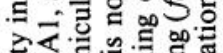

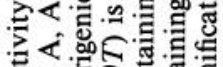

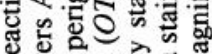

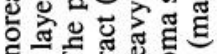

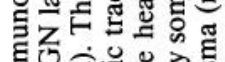

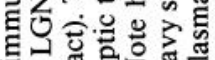

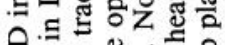
종.

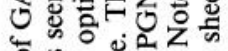
늘

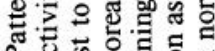

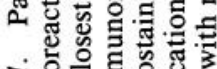

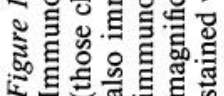



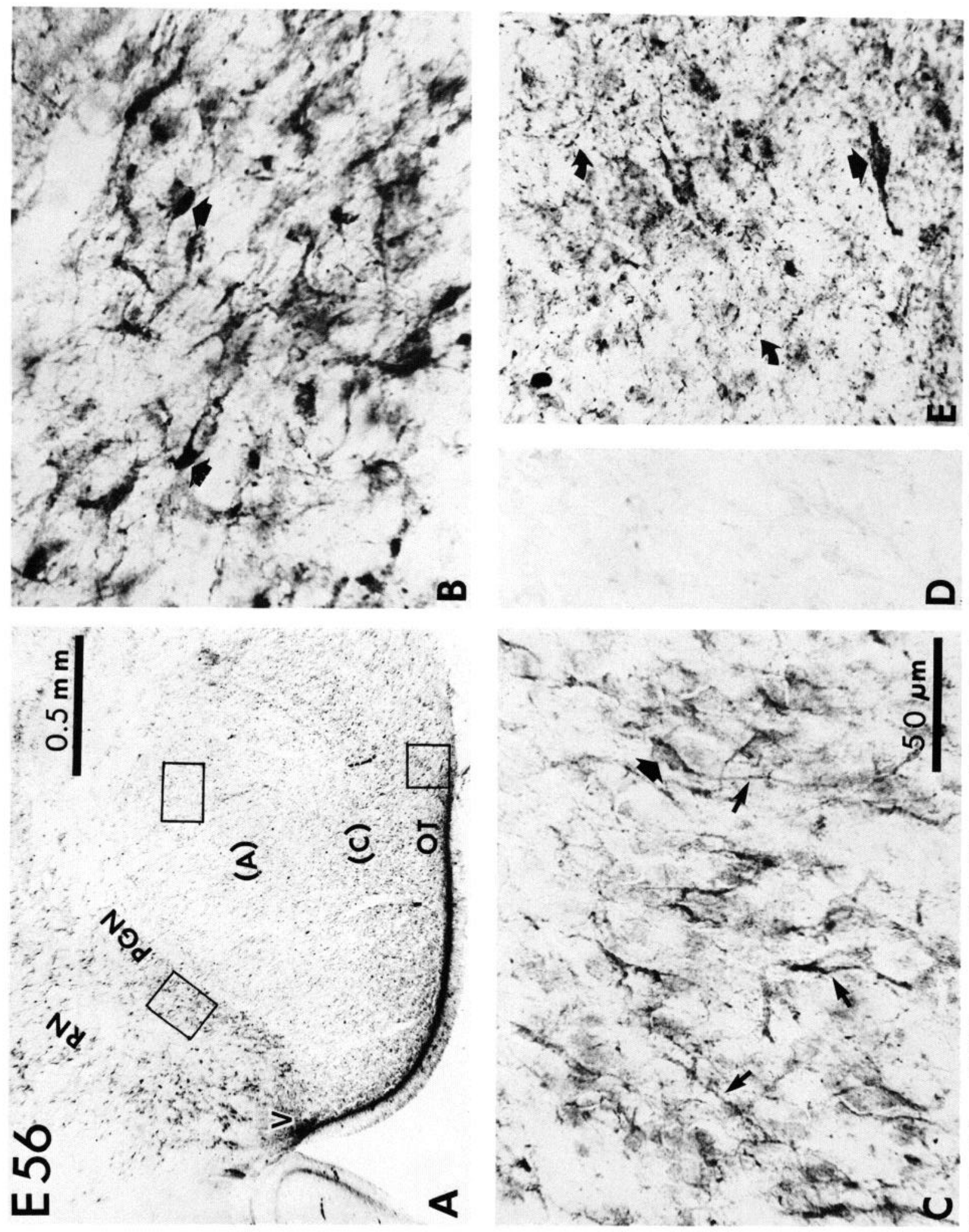

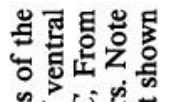
育论政

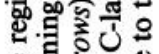

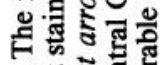

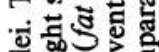

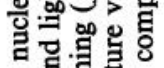
告.

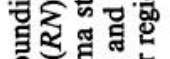
究总总 c 乙 형영

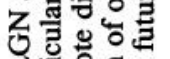
능 웅응 3. 娄

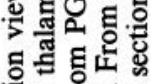

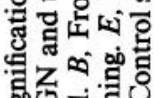

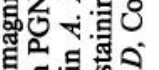
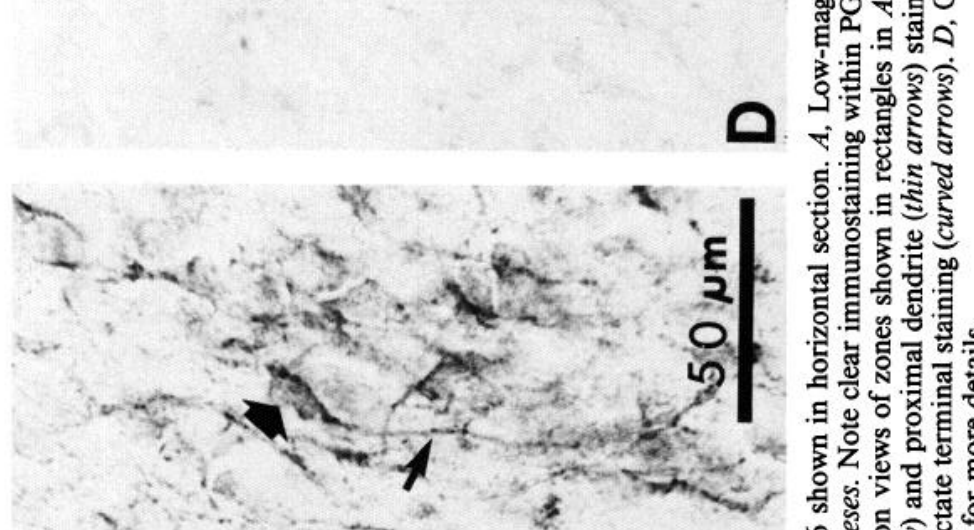

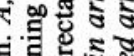
돌 局 沓

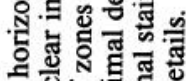
a 충

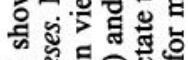

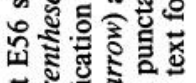

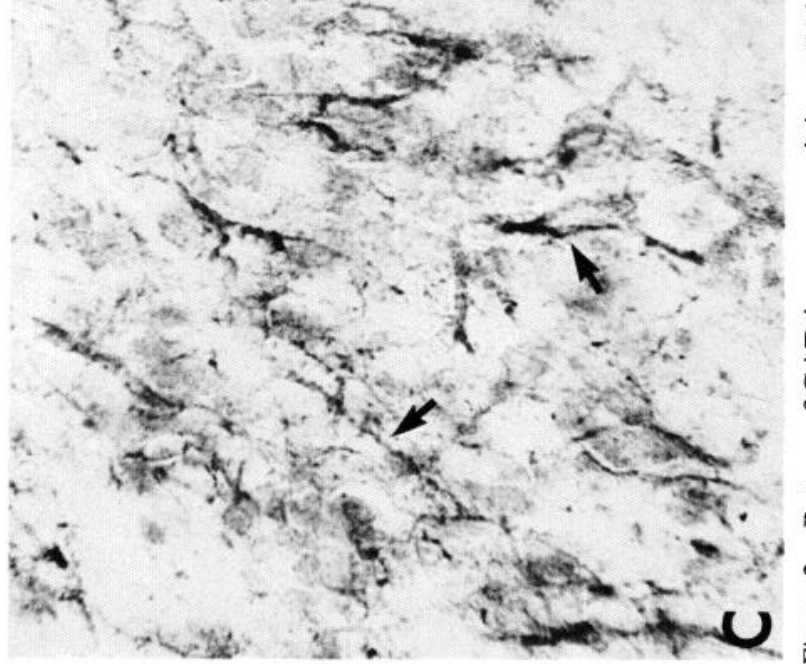

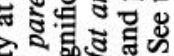
政言

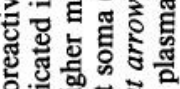

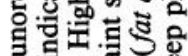
药

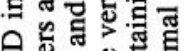
安的

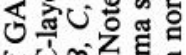

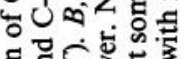
투용

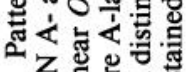

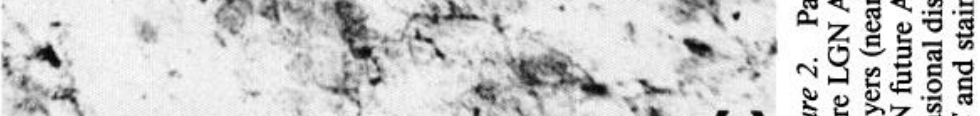

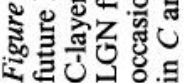




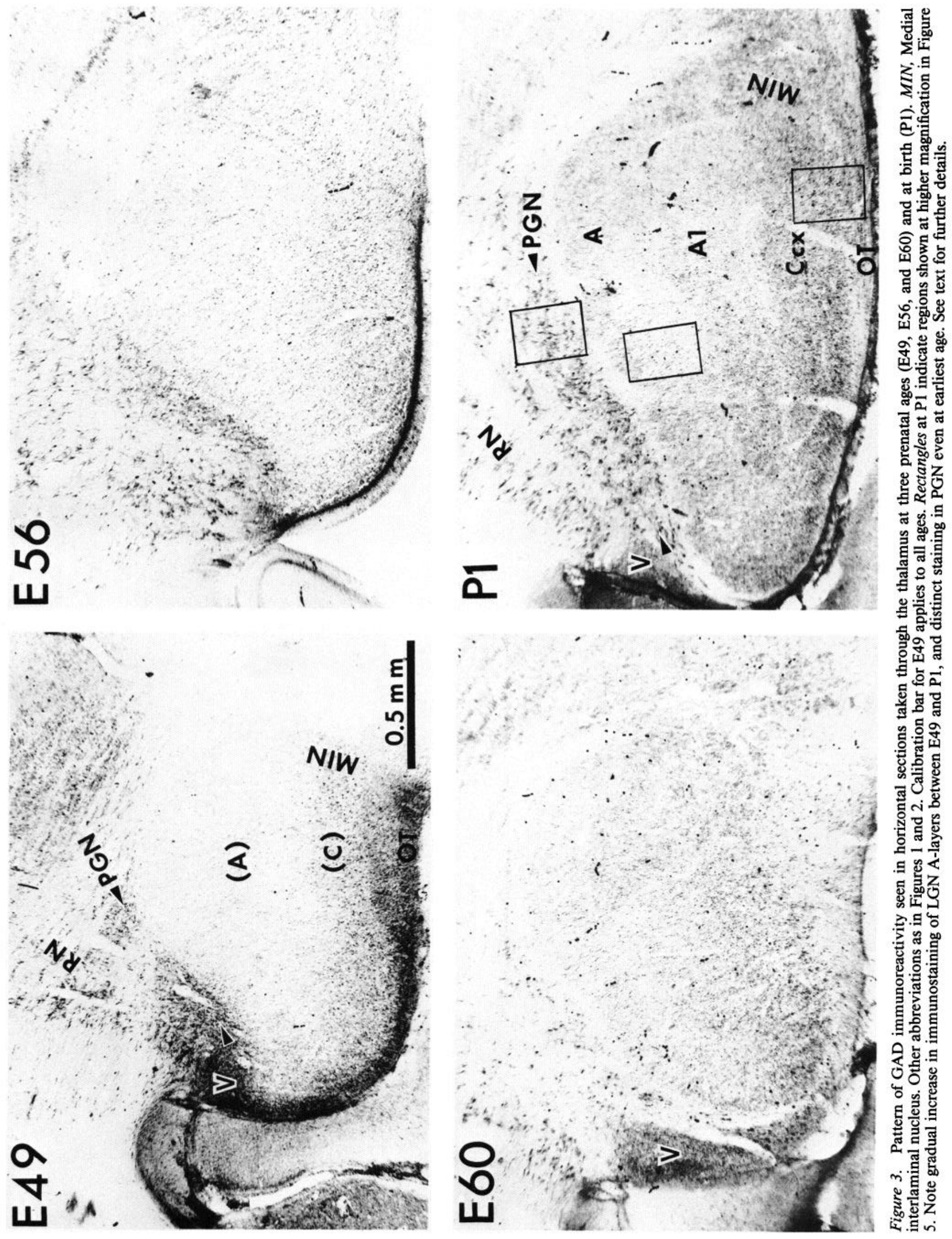


(Shatz, 1983). This segregation is completed roughly 2 weeks later at E65 (birth).

The sequence of development of GAD immunoreactivity before birth is shown in Figure 3. As at E56, the future C-layers at E49 contain a significant amount of soma and terminal stain, and the cell bodies of PGN neurons are GAD immunoreactive. In contrast to E56, the region of the future A-layers at E49 is almost free of immunoreactivity. For the reasons noted above, the staining seen in the optic tract at this age also is probably nonspecific.

In order to obtain independent confirmation that the fetal immunostaining pattern described above is indicative of the presence of GABAergic circuitry, we used an antiserum directed against another component of the GABA inhibitory pathway, an anti-GABA antibody. The application of this antiserum to sections from a postnatal case at P5O resulted in soma and terminal staining that was qualitatively similar to that seen with the GAD antiserum (compare Figs. $4 B$ and 6), although the relative intensity of terminal staining compared with soma staining was less pronounced. At E49, we found a similar agreement in the pattern of immunostaining produced using the two antibodies on adjacent sections. As shown in Figure $4 A$, GABA immunoreactivity is present in the PGN and future ventral $\mathrm{C}$-layers. While terminal staining is rare, soma staining within the PGN (Fig. $4 D$ ) and C-layers (Fig. $4 A$ ) is, if anything, more pronounced than in GAD-stained material. In fact, with GABA antiserum, soma staining can even be detected in the A-layers (Fig. 4C). (Again, at E49, sections treated with control NRS showed no staining within the PGN or LGN, and nonspecific staining of the optic tract.)

Between E49 and birth, there is a gradual increase in GAD immunostaining within the region of the future A-layers relative to the PGN (Fig. 3). The main qualitative change at birth is the appearance in the A-layers of a fine dusting of terminal staining, which can be seen at higher magnification in Figure $5 B$. Terminal staining within the C-layers is much more distinct than that seen in the A-layers (Fig. $5 \mathrm{C}$ ). Soma staining in all regions has increased in intensity (Fig. $5, A-C$ ), again with lower intensity in the A-layers relative to the C-layers. Note also the pronounced staining of dendrites in the PGN (Fig. 5A). Despite these changes, immunostaining within the LGN, particularly within the A-layers, is immature at birth.

\section{Postnatal development}

Between birth and 3 months of age, many morphological features of the LGN become mature. Dendritic branching of LGN neurons becomes progressively more extensive (Mason, 1983), the terminal arbors of retinogeniculate axons elaborate into the shapes characteristic of different morphological and functional classes (Mason, 1982; Sur et al., 1984), and retinogeniculate synapses achieve their mature ultrastructural appearance (Winfield and Powell, 1980; Mason, 1982). During this same period, GAD immunostaining gradually takes on an adultlike pattern and intensity. The sequence of changes that occur are shown in Figure 6 . By 2 weeks after birth, there has been a distinct increase in the relative intensity of terminal and soma staining within the A-layers as compared with that in the PGN and C-layers. (Compare Figs. $7 B$ and $5 B$.)

A comparison of the immunostaining seen at P14 (Fig. 7) and P35 (Fig. 8) indicates that during the intervening period there is a substantial increase in the intensity both of soma and terminal staining within the A-layers. In addition, by P35, clusters of punctate terminal staining, a prominent feature of the adult organization of GAD immunoreactivity within the A-layers, can first be detected, as shown in Figure $8 B$.

During the next 2 weeks, soma and terminal staining continue to increase in intensity, but the increase is quite gradual compared with the change that occurs between P14 and P35 (Fig.
6). The most notable change is the decrease in the apparent number of immunostained PGN cells. From about P43 onward, the PGN cells form a thin rim immediately dorsal to the LGN proper. This organization contrasts with the broad swath of PGN cells seen earlier, for example, at Pl (compare Figs. $5 A$ and $7 A$ with Figs. $1 B$ and $8 A$ ). This change in organization of the PGN is independent of the well-known developmental rotation of the LGN (Kalil, 1978; Shatz, 1983), since the plane of section was chosen to compensate for this rotation (see Materials and Methods).

By about 2 months after birth, at P63, GAD immunostaining demonstrates most of the features seen in the adult. Soma staining within the A-layers appears comparable in intensity to that within the PGN and C-layers (compare Fig. I with Figs. 6 and 9 ). Within the A-layers, distinct clusters of terminal staining are common. Perhaps the only element of organization that remains immature is found in the C-complex of the LGN. In the adult, the dorsal layer of the C-complex (layer C: Guillery, 1970) is stained more intensely than the ventral layers, as can be seen in Figure 1. However, at P63 (Fig. 9A), this difference is not apparent. In contrast, by P94 (Fig. $9 B$ ), the intensity of staining within layer $C$ has increased sufficiently to allow the layers of the C-complex to be distinguished (Fig. 6). This increase is associated with an increase in terminal staining that occurs not only in layer C, but also in layers A and Al (Fig. 9). These final changes bring about the adultlike pattern of immunostaining seen by 3 months of age.

\section{Discussion}

This study has shown that GAD immunoreactivity in the cat's LGN can be detected by E49, but the mature pattern of staining is not present until 2-3 months after birth. These conclusions rest on several assumptions pertaining to the immunohistochemical methods used here. The first is that the same protein is recognized by the GAD antibody throughout development. This assumption is supported by our finding that the patterns of GABA immunostaining at E49 and P50 are similar to those seen with the GAD antibody: The fact that both antisera give similar staining patterns at early developmental ages in particular suggests that each continues to recognize components of the same GABA-mediated inhibitory system stained in the adult. However, without direct biochemical confirmation, we cannot be certain that this antibody recognizes the same protein throughout devclopment. Along the samc lines, absence of specific staining in the one fetus studied prior to E49 does not rule out the possibility that the GAD enzyme is present in an immature form not recognized by the antibody. An alternative explanation for the absence of specific staining early on is that the concentration of the enzyme is below the level of detcctability possible with the immunohistochemical methods used here. It is conceivable that earlier specific staining might have been detected with colchicine pretreatments (Ribak et al., 1978), but concern for the likely disruptive effect of colchicine on fetal development deterred us from taking this approach.

It is worth emphasizing that the conclusions drawn here are based largely on relative differences in staining intensity between regions of LGN within the same section and, therefore, are independent of technical factors that might cause variability between experiments. For example, we considered the mature pattern of staining to be present only by P94, when staining within the dorsal layer of the C-complex (layer C) reached an intensity comparable to that seen within the A-layers of the same section. It is conceivable that the system continues to mature even beyond this age, with a further increase in staining intensity throughout the nucleus, but if so it would be hard to detect this change with the methods used here. On the other hand, it is possible to detect changes between ages in the pattern of immunostaining, such as the appearance of significant num- 


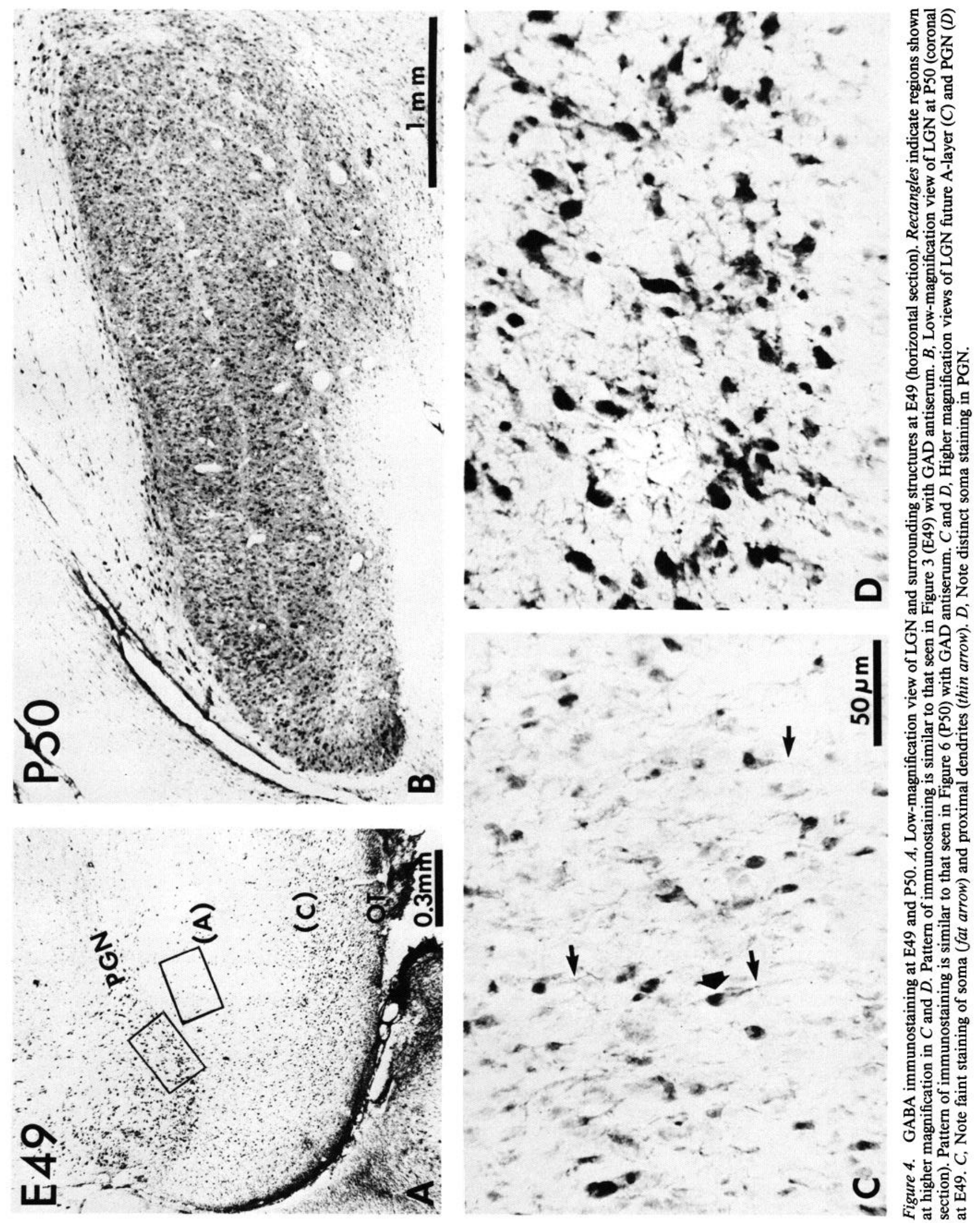



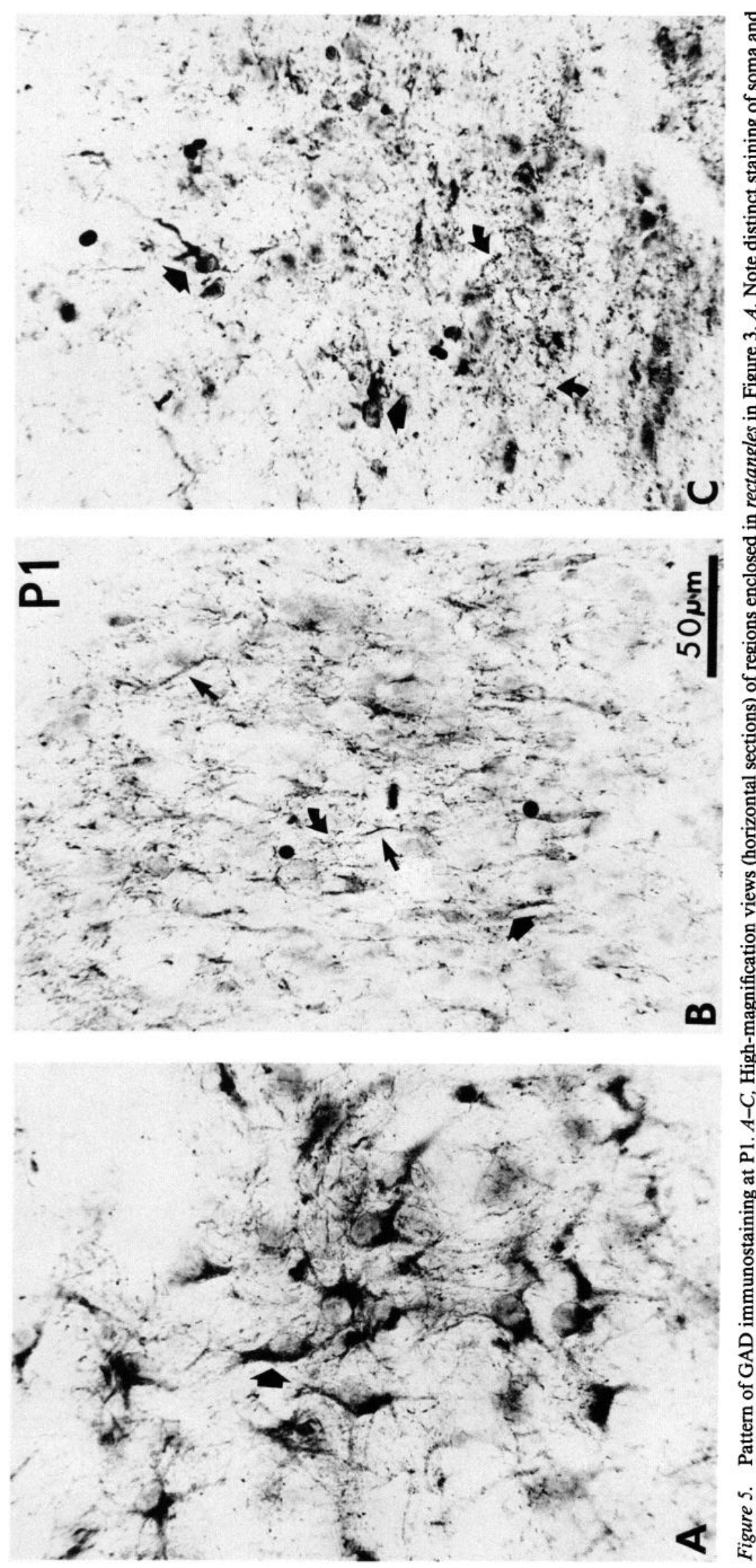

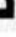
매욜 再 iz:

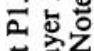
两唡

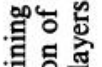
공ำ 인 要莡密 完兽 पष्ठ०

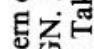

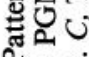
A. 实密 柁 


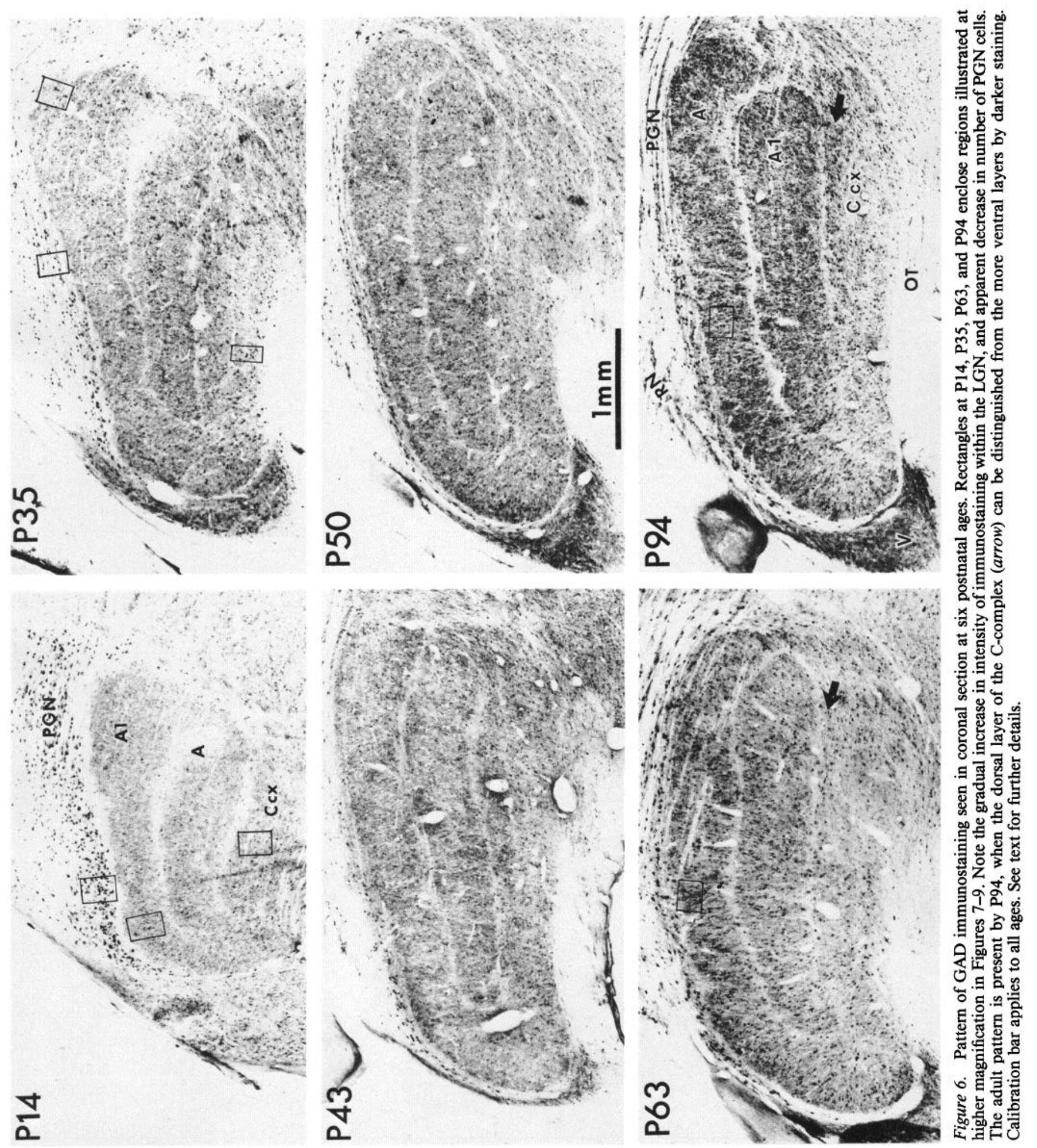



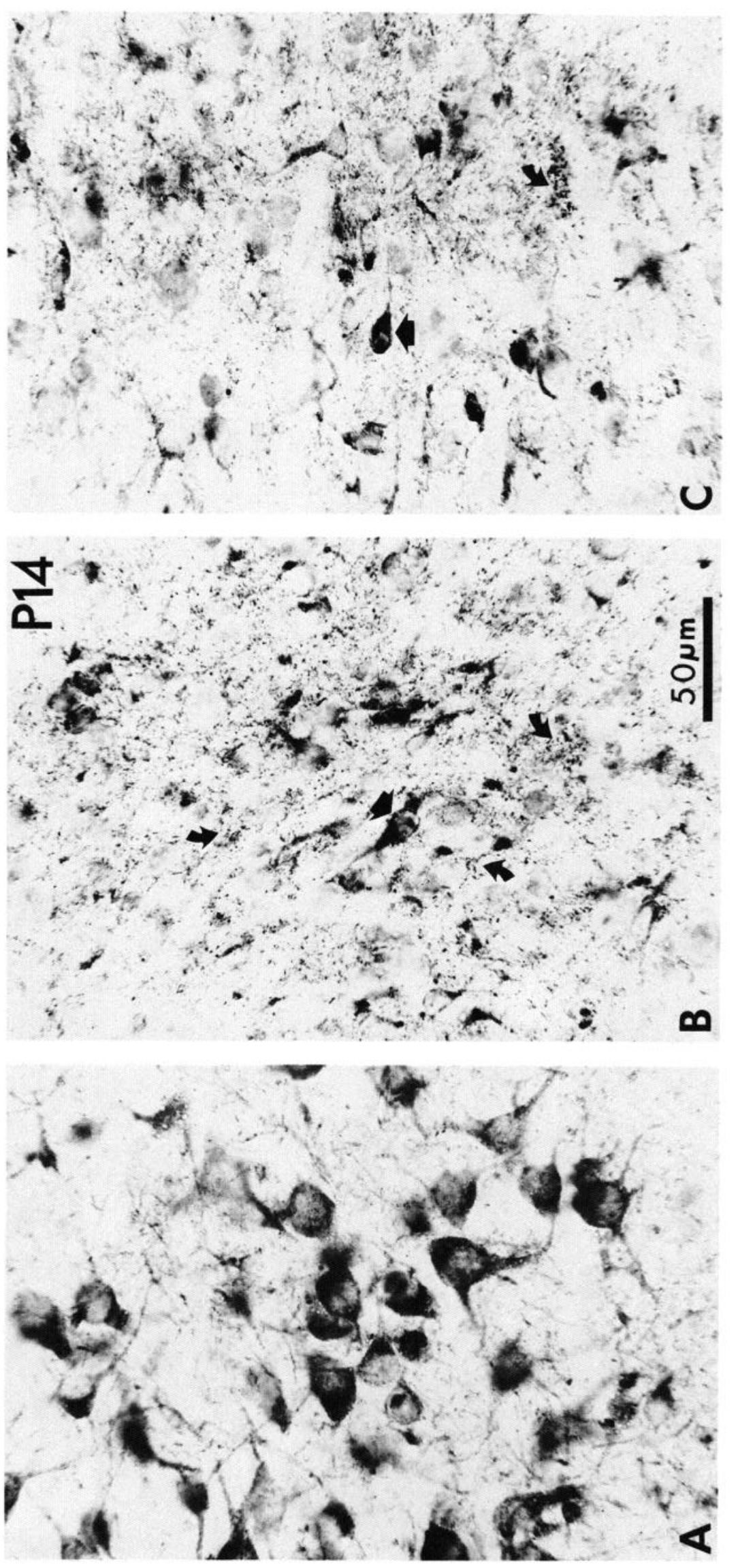

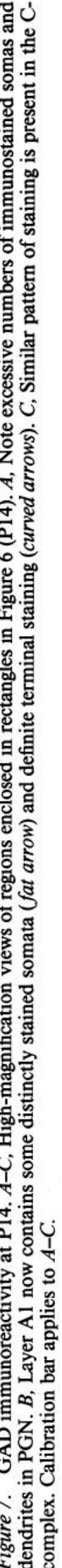



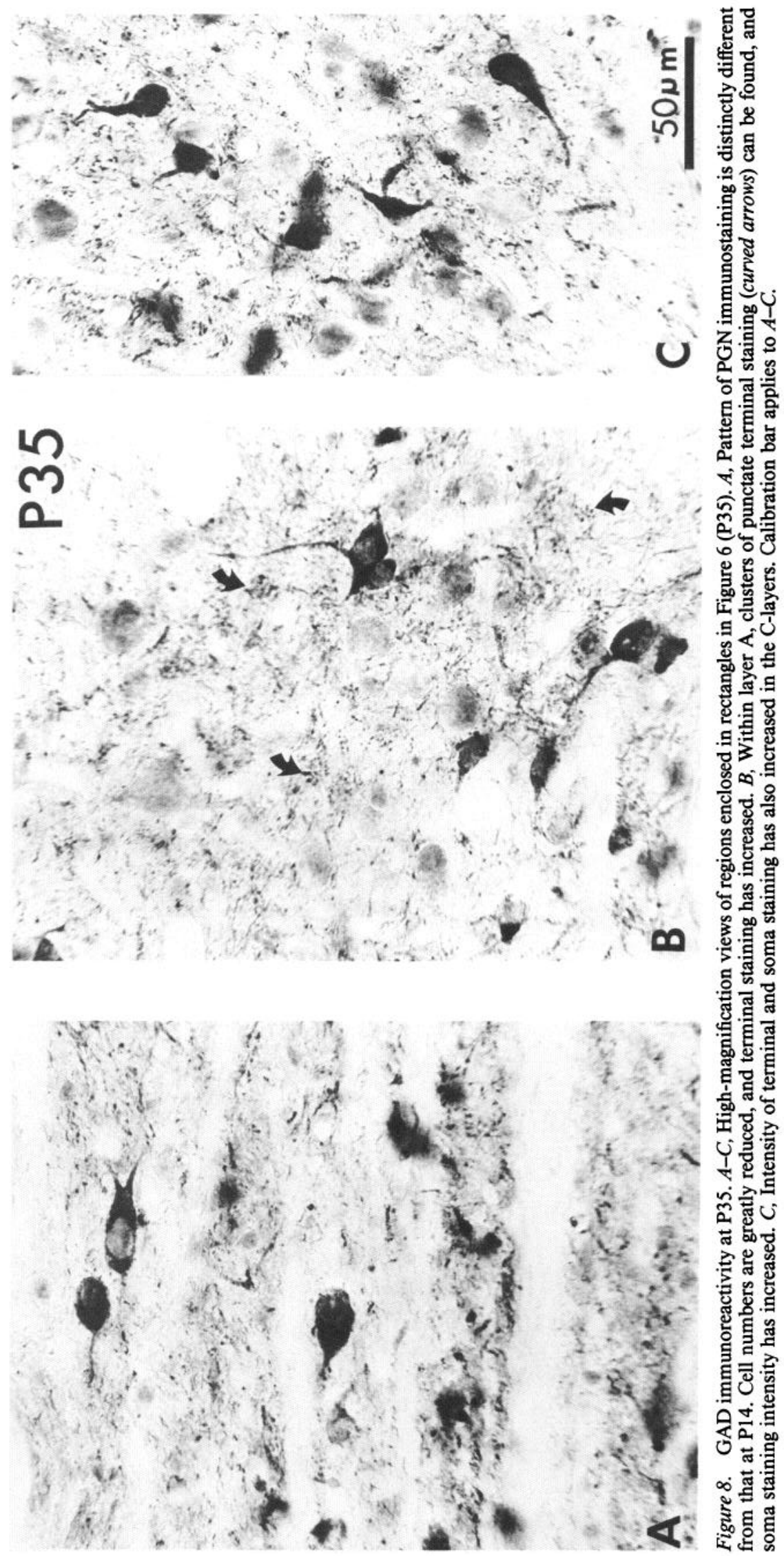

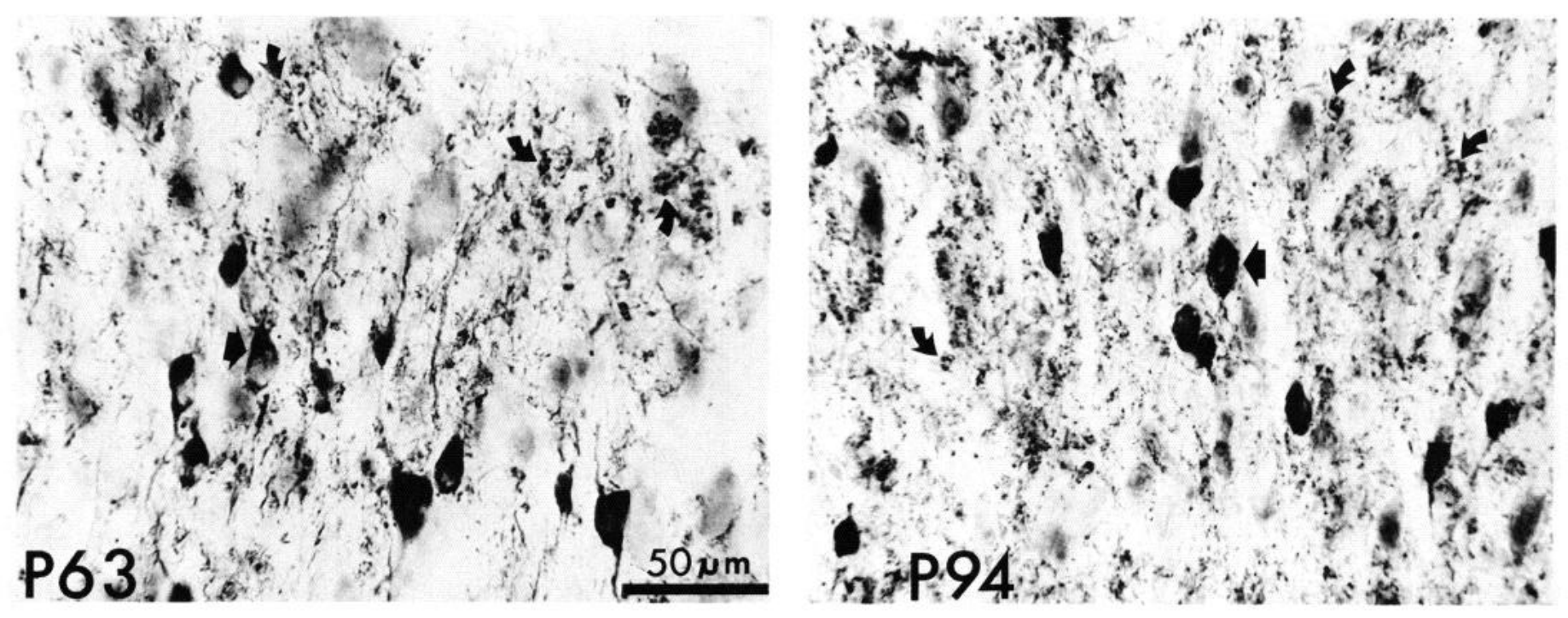

Figure 9. GAD immunoreactivity at $\mathrm{P} 63$ and P94, to show at high magnification the pattern of staining within layer A. Regions shown here are taken from areas enclosed by rectangles in Figure 6 (P63, P94). Note intense soma staining (fat arrow) and distinct and numerous clusters of punctate staining (curved arrows) at these ages. Calibration bar applies to both ages.

bers of stained somata in the A-layers and the appearance of clusters of punctate terminal staining by P35.

\section{Relation to postnatal maturation}

The pattern of GAD immunostaining requires an extended postnatal period to mature. During this same period, virtually all other aspects of LGN organization also achieve their adult properties. For instance, although the retinogeniculate axons from the two eyes are already segregated into separate layers at birth (Shatz, 1983; Sretavan and Shatz, 1986), their terminal arborizations do not achieve their final morphology until about 3 months postnatally (Sur et al., 1984). Furthermore, the LGN neurons themselves grow extensively both in soma size and in dendritic complexity postnatally, so that even by 2 months they are not quite adultlike (Friedlander et al., 1982; Kalil, 1978; Mason, 1983). At the ultrastructural level, these developmental changes are reflected in the appearance, around 2 months, of mature synaptic relations between the retinal terminals and their postsynaptic targets (Mason, 1982; Winfield and Powell, 1980). These synaptic arrangements, known as glomeruli, involve a central retinal terminal that is presynaptic both to LGN principal cells and to inhibitory interneurons whose ultrastructural appearance is characterized by profiles containing flat synaptic vesicles, F-profiles (Guillery, 1969). It is now known that the F-profiles are GAD-immunoreactive and that at the light-microscope level the contribution of the GAD-positive elements to the glomeruli takes the form of clusters of immunostained terminals (Fitzpatrick et al., 1984; Montero and Singer, 1984a, b). Thus, the finding here that clusters of GAD-immunoreactive terminals appear during the second postnatal month indicates that the maturation seen ultrastructurally is accompanied by biochemical maturation of the inhibitory interneurons.

The postnatal maturation of GAD immunoreactivity also correlates with some aspects of the maturation of the physiological response properties of LGN neurons. For example, Berardi and Morrone (1984) have studied the development of GABA-mediated inhibitory circuitry within the LGN pharmacologically. Iontophoresis of GABA and bicuculline, a GABA antagonist, does not alter visual response properties in an adultlike fashion until after 3 months of age, suggesting that some aspects of GABA-mediated inhibition do not reach functional maturity until that time. We believe that these pharmacological observations are directly complemented by our finding that the adult pattern of GAD immunostaining is attained by 3 postnatal months.

\section{Relation to prenatal development}

Microelectrode recordings from the fetal cat LGN (E39-P2) maintained in vitro have shown that the adultlike inhibitory interactions between inputs from the two eyes can first be detected in the postsynaptic responses of LGN neurons to electrical stimulation of the optic nerves as early as E59, although in only about $6 \%$ of the cells (Shatz and Kirkwood, 1984). By birth, roughly $50 \%$ of the cells show binocular inhibitory interactions. The results of the present study indicate that the appearance of inhibitory interactions correlates with the appearance of immunostaining within the A-layers. Even at this age, LGN immunostaining is faint; by birth, terminal and soma staining, particularly within the A-layers, have become more distinct. These correlations suggest that the appearance and progressive development of binocular inhibitory interactions may be a direct consequence of the onset of maturation of the intrinsic inhibitory LGN neurons themselves.

Another possibility is that the inhibitory interactions seen during the fetal period are mediated principally by neurons of the PGN and ventral C-layers. In the adult, PGN neurons are known to project into the LGN as part of the GABA-mediated inhibitory circuitry driven by collaterals of LGN neurons (Ahlsen and Lindstrom, 1982; Dubin and Cleland, 1977; Friedlander et al., 1981; Lindstrom, 1982). This possibility is quite likely in view of our finding here that GAD immunostaining of the PGN and future ventral C-layers appears remarkably early in development, at E49 or perhaps even earlier. Thus, it may be that the first inhibitory interactions seen within the LGN involve the neurons of the PGN, with the major portion of the intrinsic interneuron-supplied inhibition arising later, around birth.

It is worth noting that GAD immunostaining in the PGN and ventral C-layers is detected earlier (E49) than inhibitory interactions in the LGN elicited by optic nerve stimulation (E59: Shatz and Kirkwood, 1984). This disparity could arise for a variety of reasons. For example, at E49, the terminals of these neurons may not be present within the LGN, or, if present, they 
might contain the synthetic enzymc for GABA but not produce sufficient quantities of transmitter for release, the machinery for release may not be mature, or postsynaptic GABA receptors may not be present at these early ages. Another possibility that cannot be discounted at this point is that even if GABA is present in a relcasable form, during early development it may not act as a conventional neurotransmitter, but rather may have some trophic role.

Throughout this discussion, we have assumed that, as in the adult, the presence of GAD immunoreactivity during development signals the existence of inhibitory inputs to the postsynaptic LGN neurons. In this context, it is worth noting that in at least one system, the developing rabbit hippocampus, GABA has been shown to depolarize initially, and only later in development to hyperpolarize pyramidal neurons studied in vitro (Mueller et al., 1984). It is not yet clear whether the early depolarization is associated with a conductance increase; if so, the depolarization could still produce inhibition by holding the membrane potential below the threshold for production of an action potential. Nevertheless, we wish to emphasize that, until at least E59, there exists no physiological evidence for the presence of inhibitory interactions.

\section{Sequence of maturation}

The onset of GAD immunostaining in the LGN/PGN complex varied dramatically between regions, with the $P G N$ and ventral portion of the C-layers showing definitive soma staining by E49, followed by the A-layers, where definitive soma staining does not appear until shortly after birth. Tritiated thymidine studies of LGN development in the cat have shown that timing of neurogenesis per se cannot be responsible for this sequence of maturation. The first neurons generated are destined for the PGN, while the very last are destined for the C-layers; however, during the ensuing period, neurons destined for all layers of the LGN and PGN can have the same birth date (Hickey and Hitchcock, 1984; Hitchcock et al., 1984; Shatz, 1981). Thus, the spatiotemporal pattern of neurogenesis cannot entirely account for the sequence of immunostaining in view of the fact that the ventral portion of the $C$-layers and the optic tract show early immunoreactivity.

An alternative might be that the appearance of immunostaining is correlated with some other aspect of maturation. For example, the C-layers of the LGN are the first to be invaded by afferents from the retina (Shatz, 1983). In both cat and ferret, many cells within the C-layers also appear morphologically mature earlier than their counterparts in the A-layers (Linden et al., 1981; Shatz, 1983). Thus, the GAD immunoreactivity seen here may be another reflection of the early maturation of the C-layers.

The early GAD immunostaining of the PGN, on the other hand, may be correlated with the presence of afferent inputs from the brain stem and cortex, as mentioned above. For the corticogeniculate afferents, it is very likely that during development in the cat, as in the monkey (Shatz and Rakic, 1981), they accumulate just above the LGN in the vicinity of the PGN and thalamic radiations, prior to their ingrowth into the LGN proper. In this context, it is interesting to note that an excessive number of GAD-positive PGN neurons arc present during fetal and early postnatal ages. This observation is consistent with that of Murakami (personal communication), who also has remarked on the excessive numbers of neurons in the cat's PGN during the first 2 months of postnatal life. Thus, it may be that the initial population of GAD-positive PGN neurons serves as a temporary target for the corticogeniculate afferents, and that the decrease in number of PGN neurons seen postnatally is a consequence of the ingrowth of the afferents into the LGN proper.

\section{Relation to critical period for monocular deprivation}

Until recently, the cat's retinogeniculate system was assumed to be rather mature during early postnatal life, and therefore relatively immune to the effects of abnormal visual experience. However, as mentioned in the beginning of this discussion, many morphological features of retinogeniculate axons and physiological properties of LGN neurons do not reach maturity until about 3 months after birth. This raises the possibility that this part of the visual system, in addition to visual cortex, may be susceptible to modification by experience. Recent evidence shows that this is indeed the case: Abnormal visual experience in the cat has been shown to alter the response properties of LGN neurons (for review, see Sherman and Spear, 1982) and to affect the morphology of retinogeniculate axons (Sur et al., 1982). Our finding here that GAD immunoreactivity is not adultlike until 3 months postnatally raises the possibility that abnormal experience could affect the maturation of the GABA inhibitory system as well. If so, the A-layers might be more sensitive than the ventral C-layers, in view of our finding that immunostaining of the ventral C-layers appears more developmentally advanced by birth. This suggestion is consistent with the observation of Hickey (1980) that monocular deprivation affects soma size in the A-layers, but not in the ventral C-layers. Similar considerations may apply to the primate visual system, in view of the recent report by Ogren et al. (1983) that GAD immunoreactivity in monkey LGN also is immature at birth. Thus, it may be that some of the abnormal response properties seen in LGN neurons and at the level of the visual cortex subsequent to visual deprivation derive from altered inhibitory interactions within the LGN.

\section{References}

Ahlsen, G., and S. Lindstrom (1982) Excitation of perigeniculate neurones via axon collaterals of principal cells. Brain Res. 236: 477-481.

Ariel, M., and N. W. Daw (1982) Pharmacological analysis of directionally sensitive rabbit retinal ganglion cells. J. Physiol. (Lond.) 324 : $161-185$.

Berardi, N., and M. C. Morrone (1984) Development of gammaaminobutyric acid mediated inhibition of $\mathrm{X}$ cells of the cat lateral geniculate nucleus. J. Physiol. (Lond.) 357: 525-537.

Blakemore, C., and R. C. Van Sluyters (1974) Reversal of the physiological effects of monocular deprivation in kittens: Further evidence for a sensitive period. J. Physiol. (Lond.) 237: 195-216.

Burke, W., and A. J. Sefton (1966) Inhibitory mechanisms in lateral geniculate nucleus of rat. J. Physiol. (Lond.) 187: 231-246.

Dubin, M. W., and B. G. Cleland (1977) Organization of visual inputs to interneurons of lateral geniculate nucleus of the cat. J. Neurophysiol. 40: 410-427.

Famiglietti, E. V., and A. Peters (1972) The synaptic glomerulus and the intrinsic neurons in the dorsal lateral geniculate nucleus of the cat. J. Comp. Neurol. 144: 285-334.

Fitzpatrick, D., G. R. Penny, and D. E. Schmechel (1984) Glutamic acid decarboxylase-immunoreactive neurons and terminals in the lateral geniculate nucleus of the cat. J. Neurosci. 4: 1809-1829.

Friedlander, M. J., C.-S. Lin, L. R. Stanford, and S. M. Sherman (1981) Morphology of functionally identified neurons in lateral geniculate nucleus of the cat. J. Neurophysiol. 46: 80-129.

Friedlander, M. J., K. A. C. Martin, and C. Vahle-Hinz (1982) The postnatal development of structure of physiologically identified retinal ganglion ccll (r.g.c.) axons in the kitten. J. Physiol. (Lond.) 336: 28-29P.

Guillery, R. W. (1969) The organization of synaptic interconnections in the laminae of the dorsal lateral geniculate nucleus of the cat. $Z$. Zellforsch. Mikrosk. Anat. 96: 1-38.

Guillery, R. W. (1970) The laminar distribution of retinal fibers in the dorsal lateral geniculate nucleus of the cat: A new interpretation. J. Comp. Neurol. 138: 339-368.

Hickey, T. L. (1980) Development of the dorsal lateral geniculate nucleus in normal and visually deprived cats. J. Comp. Neurol. 189: 467-481. 
Hickey, T. L., and P. F. Hitchcock (1984) Genesis of neurons in the dorsal lateral geniculate nucleus of the cat. J. Comp. Neurol. 228. 186-199

Hitchcock, P. F., T. L. Hickey, and C. G. Dunkel (1984) Genesis of morphologically identified neurons in the dorsal lateral geniculate nucleus of the cat. J. Comp. Neurol. 228: 200-209.

Hubel, D. H., and T. N. Wiesel (1970) The period of susceptibility to the physiological effects of unilateral eye closure in kittens. J. Physiol. (Lond.) 206: 419-436.

Kalil, R. (1978) Development of the dorsal lateral geniculate nucleus in the cat. J. Comp. Neurol. 182: 265-292.

Kato, H., M. Yamamoto, and H. Nakahama (1971) Intracellular recordings from the lateral geniculate neurons of cats. Jpn. J. Physiol. 21: 307-323.

Linden, D. C., R. W. Guillery, and J. Cucchiaro (1981) The dorsal lateral geniculate nucleus of the normal ferret and its postnatal development. J. Comp. Neurol. 203: 189-211.

Lindstrom, S. (1982) Synaptic organization of inhibitory pathways to principal cells in the lateral geniculate nucleus of the cat. Brain Res. 234: 447-453.

Mason, C. A. (1982) Development of terminal arbors of retino-geniculate axons in the kitten. II. Electron microscopical observations. Neuroscience 7: 561-582.

Mason, C. A. (1983) Postnatal maturation of neurons in the cat's lateral geniculate nucleus. J. Comp. Neurol. 217: 458-469.

McLean, I. W., and P. K. Nakane (1974) Periodate-lysine-paraformaldehyde fixative: A new fixative for immunoelectron microscopy. J. Histochem. Cytochem. 22: 1077-1083.

Montero, V. M., and W. Singer (1984a) Ultrastructure and synaptic relations of neural elements containing glutamic acid decarboxylase (GAD) in the perigeniculate nucleus of the cat. Exp. Brain Res. 56: 115-125.

Montero, V. M., and W. Singer (1984b) Ultrastructure and synaptic relations of glutamic acid decarboxylase $(\mathrm{G} \Lambda \mathrm{D})$-immunoreactive neural elements in the dorsal lateral geniculate nucleus of the cat. Neurosci. Abstr. 10: 55.

Mueller, A. L., J. S. Taube, and P. A. Schwartzkroin (1984) Development of hyperpolarizing inhibitory postsynaptic potentials and hyperpolarizing response to gamma-aminobutyric acid in rabbit hippocampus studied in vitro. J. Neurosci. 4: 860-867.

Oertel, W. H., D. E. Schmechel, E. Mugnaini, M. L. Tappaz, and I. J. Kopin (1981) Immunocytochemical localization of glutamate decarboxylase in rat cerebellum with a new antiserum. Neuroscience 6 : 2715-2735.

Ogren, M. P., A. E. Hendrickson, and J.-Y. Wu (1983) Neonatal development of GAD immunoreactivity and GAD + synapses in the dorsal lateral geniculate of the monkey: A light and electron microscopic analysis. Neurosci. Abstr. 9: 25.

Ribak, C. E., J. E. Vaughn, and K. Saito (1978) Immunocytochemical localization of glutamic acid decarboxylase in neuronal somata following colchicine inhibition of axonal transport. Brain Res. 140:315332.

Shatz, C. J. (1981) Inside-out pattern of neurogenesis of the cat's lateral geniculate nucleus. Neurosci. Abstr. 7: 140.

Shatz, C. J. (1983) The prenatal development of the cat's retinogeniculate pathway. J. Neurosci. 3: 482-499.

Shatz, C. J., and P. A. Kirkwood (1984) Prenatal development of functional connections in the cat's retinogeniculate pathway. J. Neurosci. 4: 1378-1397.

Shatz, C. J., and P. Rakic (1981) The genesis of efferent connections from the visual cortex of the fetal rhesus monkey. J. Comp. Neurol 196: 287-307.

Sherman, S. M., and P. D. Spear (1982) Organization of visual pathways in normal and visually deprived cats. Physiol. Rev. 62: 738855 .

Shotwell, S. L., M. B. Luskin, and C. J. Shatz (1984) Development of GAD immunoreactivity correlates with onset of inhibition in the cat's lateral geniculate nucleus. Neurosci. Abstr. 10: 142.

Sillito, A. M. (1977) Inhibitory processes underlying the directional specificity of simple, complex and hypercomplex cells in the cat's visual cortex. J. Physiol. (Lond.) 271: 699-720.

Sillito, A. M., and J. A. Kemp (1985) The influence of GABAergic inhibitory processes on the receptive field structure of $X$ and $Y$ cells in cat LGN. Brain Res. 277: 63-77.

Singer, W., and N. Bedworth (1973) Inhibitory interaction between $\mathrm{X}$ and $\mathrm{Y}$ units in the cat lateral geniculate nucleus. Brain Res. 49. 291-307.

Sretavan, D. W., and C. J. Shatz (1986) Prenatal development of retinal ganglion cell axons: Segregation into eye-specific layers within the cat's lateral geniculate nucleus. J. Neurosci. 6: 234-251.

Sur, M., A. L. Humphrey, and S. M. Sherman (1982) Monocular deprivation affects $\mathrm{X}$ - and $\mathrm{Y}$-cell retinogeniculate terminations in cats. Nature 300: 183-185.

Sur, M., R. E. Weller, and S. M. Sherman (1984) Development of Xand Y-cell retinogeniculate terminations in kittens. Nature 310:246249.

Suzuki, H., and E. Kato (1966) Binocular interaction at cat's lateral geniculate body. J. Neurophysiol. 29: 909-920.

Winfield, D. A., and T. P. S. Powell (1980) An electron-microscopical study of the postnatal development of the lateral geniculate nucleus in the normal kitten and after eyelid suture. Proc. $\mathrm{R}$. Soc. London [Biol.] 210: 197-210. 